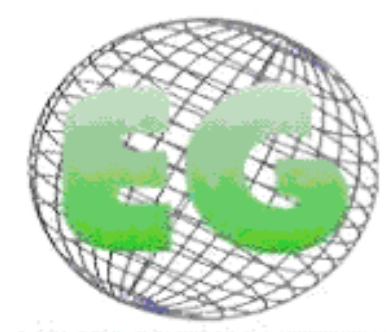

ISSN 1695-6141 N 27

\title{
Teorías y modelos en la práctica enfermera. ¿Un binomio imposible?
}

\author{
Theories and models in clinical nursing practice. An impossible relationship?
}

\section{*Sancho Cantus, D., "*Prieto Contreras, L.}

\author{
*Facultad de Enfermería de la Universidad Católica de Valencia San Vicente Mártir. E-mail: \\ dsanch46@hotmail.com
}

Palabras clave: enfermería; teorías y modelos; práctica clínica; desarrollo

Keywords: nursing; theories and models; clinical practice; development

\section{RESUMEN}

Introducción. En las últimas décadas la Enfermería española ha sufrido numerosos cambios que han contribuido en mayor o menor medida al desarrollo de la disciplina. No obstante sigue existiendo en la actualidad una barrera que dificulta y retrasa este desarrollo: la ecuación compuesta por teoría y práctica.

Desarrollo del tema. Son diversos los factores que influyen en la retroalimentación entre estos dos elementos, y que repercuten en el crecimiento que experimenta la propia Enfermería. Estamos en la actualidad en un periodo de inflexión en el que es necesario imbricar de una forma efectiva estas dos vertientes, y todo ello con un doble objetivo: aplicar a la práctica diaria los modelos teóricos existentes y disponibles, y fundamentar las decisiones clínicas en evidencias científicas que respalden nuestras actuaciones. Con todo ello se pretende que la Enfermería utilice su corpus propio de conocimientos y sea capaz de nutrirse y de aprovecharlos para su desarrollo en todos sus ámbitos competenciales.

Conclusiones: En estos momentos la Enfermería se halla inmersa en un complejo proceso de reconceptualización de la propia disciplina, y se abre un abanico de posibilidades que deberán ir acompañadas inevitablemente de la asunción de ciertos desafíos para los cuales será necesario estar correctamente formados.

\section{ABSTRACT}

Introduction: In the last few decades, Spanish Nursing has suffered several changes that have contributed to a lesser or greater extent to the development of this discipline. However, today there is still a barrier that hinders and delays this development: the equation consisting of theory and practice.

Development of the subject. There are several factors which influence the feedback between these two elements and have an impact on the growth that nursing itself undergoes. Nowadays, we are in a period of inflection in which it is necessary to combine both aspects with a dual objective: to put into daily practice the current theoretical models which are available and to base the clinical decisions on scientific evidence which 
can support our proceedings. Moreover, it is intended that nursing uses its own corpus of knowledge to learn and profit from it in order to develop in all its areas.

Conclusions: Nowadays, nursing is immersed in a complex process of conceptualization of its own discipline. There is a wide range of possibilities which must inevitably be accompanied by the acceptance of certain challenges for which individuals must be trained.

\section{INTRODUCCIÓN}

Solemos definir a la Enfermería como una ciencia, en el sentido de adquisición de conocimientos mediante la observación y la experimentación, y la práctica basada en la evidencia científica. Asimismo se la considera un arte y una filosofía, compatibles con esta concepción científica ${ }^{(1,2)}$.

Existe una relación directa e inmediata entre el binomio teoría/práctica, y es esta una cuestión que está siendo ampliamente debatida en la actualidad como punto de referencia para situar a la Enfermería epistemológicamente ${ }^{(2,3)}$. En el desarrollo de nuestra profesión existen factores relacionados con la teoría y la práctica que se revelan problemáticos y precisan ser reflexionados, como, por ejemplo, la separación crónica entre estos dos aspectos (algunos autores ${ }^{(4,5)}$ hablan de divorcio entre conocimiento y práctica), y la realidad frente al ideal de la Enfermería. En este sentido resulta imprescindible unificar teoría y práctica para poder fundamentarse ambas en la realidad objetiva y en las evidencias científicas. En la actualidad son muchos los autores que defienden una apuesta por la práctica enfermera considerándola una pieza clave junto con la teoría para el desarrollo enfermero ${ }^{(4-6)}$. La separación entre teoría y práctica, entre sujeto investigador y sujeto investigado produce un efecto descontextualizador que inhabilita las acciones desde el punto de vista de la eficacia esencial ${ }^{(7)}$.

Existe una falta de concisión epistemológica en la Enfermería que da lugar al solapamiento o a la incorrecta ubicación de la disciplina en la taxonomía científica. El principal reto que se nos presenta, la consolidación profesional y científica, está íntimamente relacionado con la indefinición epistemológica y la adopción de métodos que contradicen la esencia del objeto de la disciplina ${ }^{(8,9)}$.

La Enfermería Basada en la Evidencia (EBE) nos hace reflexionar sobre los referentes epistemológicos y metodológicos en los que se basa la práctica clínica habitual. Collière ${ }^{(1)}$ afirmaba: "dime qué conocimientos aplicas y te diré qué clase de cuidados das". Se hace necesaria la búsqueda de escenarios de reflexión en torno al colectivo enfermero y las instituciones donde desarrollan su labor profesional. No obstante, el conseguir transformar la investigación en práctica es una tarea exigente que requiere rigor intelectual y disciplina, creatividad, juicio clínico, destreza, inteligencia organizacional y paciencia ${ }^{(10)}$.

\section{DESARROLLO DEL TEMA}

Inicialmente podríamos interrogarnos sobre el por qué de la necesidad de las teorías y modelos para la Enfermería como paso previo para la aplicación de éstos en la práctica. La teoría proporciona una base científica de conocimientos para poder llevar a la práctica nuestro trabajo, nos fundamenta y nos enseña a actuar con sentido. Es capaz de describir, explicar, predecir y controlar los fenómenos ${ }^{(11)}$. Profundizando sobre la cuestión se observa que la teoría ayuda a analizar los hechos, además de potenciar y adecuar la práctica de los cuidados y la investigación de enfermería. 
La profesora Zabalegui( ${ }^{(2)}$ indica que "los hechos que no se pueden comprobar experimentalmente, no son considerados como hechos teóricos", por lo que aquellos aspectos cualitativos del concepto de salud, de persona, como actitudes o valores, son desechados de inmediato al no ser cuantificables ni medibles. La solución al conflicto apunta hacia una coordinación entre teóricos y clínicos, haciendo partícipes a los clínicos del desarrollo conceptual con el objeto de reducir la separación entre ambos campos. La experiencia también es necesaria para reinterpretar los hallazgos y modificar o reestructurar las teorías, por lo que se insiste en la necesidad de que las teorías de enfermería deben intentar integrar conocimientos y práctica con dos finalidades:

* Implicar al mayor número de profesionales en el desarrollo de un cuerpo de conocimientos propio de la disciplina

* Eliminar la distancia entre lo que se aprende en las universidades y lo que se vive en la praxis diaria

Afaf Meleis, durante el VII Coloquio Panamericano de Investigación en Enfermería que tuvo lugar en Bogotá en el año 2000 (12), defendió la idea de que hasta que la Enfermería no consiga armonizar teoría y práctica, seguirán observándose fisuras en la base científica del conocimiento de esta disciplina.

Cuando nos disponemos a valorar la situación de salud de un sujeto, nos encontramos modelos que tratan la interacción entre el sujeto y su entorno (Orlando, Kim), modelos evolucionistas centrados en la teoría del desarrollo (Peplau), modelos de necesidades humanas (Henderson, Rogers, Orem) o modelos de sistemas (Roy, Newman) ${ }^{(12-14)}$. En la etapa diagnóstica aparecen distintas taxonomías: NANDA, OMAHA... En la ejecución del Plan de Cuidados propiamente dicha tenemos otras taxonomías: NOC, CIPE para los criterios de resultados, NIC, proyecto SIGNO...

Desde el punto de vista teórico, esta variedad de taxonomías para organizar el cuerpo de conocimientos engrandece a la disciplina, pero en la práctica real y diaria puede suponer cierta confusión que comporta en última instancia la mala utilización de estas herramientas o incluso la no utilización de las mismas. Se necesita el lenguaje que refleje la práctica, se necesita la práctica que formule la teoría y se necesita la filosofía que orienta la dirección de los cuidados.

La profesora Sanjuán (15) describe algunos interrogantes que surgen en la relación entre teoría y práctica, como son:

¿La práctica constituye un marco adecuado para el aprendizaje experiencial?

El aprendizaje experiencial, ¿posibilita el desarrollo profesional y la innovación?

¿Qué base teórico-práctica necesita un futuro profesional para poder asentar sobre ella, a medida que avanza por su trayectoria, nuevos conocimientos, nuevas habilidades, pero también nuevas formas de comprender su función cuidadora?

Para Schön ${ }^{(16)}$ la práctica es un espacio de privilegio que permite el aprendizaje experiencial, aprendizaje que se produce por la integración de la experiencia tras un periodo de reflexión. Este aprendizaje se caracteriza por ser interpretativo individual, no generalizable e implícito. El marco que posibilita este tipo de aprendizajes se denomina investigación acción, que se resume como un proceso de reflexión en la práctica ${ }^{(17-19)}$. De esta forma se puede considerar que el profesional de enfermería competente es aquel que utiliza sus conocimientos, habilidades y actitudes para emitir juicios clínicos y solucionar problemas, 
conjuntando para ello las bases teóricas de la enfermería con su aplicación a la práctica. Por tanto, lo que las enfermeras hacen se basa en lo que las enfermeras conocen.

La excelencia de los cuidados precisa de marcos de referencia, de profesionales con voluntad de implicarse, de valores y del poder para llevar a término los cambios ${ }^{(20)}$. $Y$ en eso precisamente está inmersa la Enfermería de principios del siglo XXI.

\section{ANÁLISIS DE LA SITUACIÓN. ¿CÓMO ESTÁ LA ENFERMERÍA?}

Si la ciencia utiliza la investigación para la producción de nuevos conocimientos, teorías, a su vez la investigación es precedida por enfoques paradigmáticos. En la evolución histórica de la investigación en Enfermería, Polit y Hunter [19] registraron su aparición en 1859 con los apuntes de enfermería de Florence Nightingale, quien analizó datos sobre factores ambientales que favorecen el medio físico y emocional, contribuyendo así a la configuración de aspectos cuantitativos a partir de sustantivos o cualitativos, como el ambiente o el bienestar. Entre 1900-1923 el Comité de Estudios para la Educación de Enfermería en EEUU facilita el informe Golmark [19], evidencia histórica de observación e investigación. En este decenio de 1900 se impulsa la investigación en enfermería con el Centro de Investigaciones en Enfermería en el Walter Reed Army Institute of Research, entre otros. La investigación en Enfermería tomó durante este lapso de tiempo un giro trascendental: las enfermeras empezaron a investigar sobre sí mismas, quién es la Enfermería, qué hace... Liderman $[19,20]$ registró el cambio de orientación en la investigación; se crea conciencia de generar base científica para la práctica. Se tiene el convencimiento que desde la teoría se generan bases científicas para la práctica. Actualmente existe el convencimiento, además, de que desde la práctica también se sientan bases para el conocimiento y la transformación de la misma.

¿Cuál es uno de los mayores desafíos a los que se enfrenta nuestra disciplina? Reforzar la base de conocimientos para la práctica de la disciplina. Enfermería se enfrenta ahora al reto de generar sus propios conocimientos a través de la investigación y construir su realidad desde su propia perspectiva, es decir, su práctica y objeto disciplinar, que es el cuidado [10]. Es frecuente en distintas disciplinas el uso de la matriz DAFO cuyo objetivo es explorar cuál es el estado de una situación, analizando para ello las Debilidades, Amenazas, Fortalezas y Oportunidades. En Enfermería es un concepto prácticamente inédito, puesto que no se suele utilizar para efectuar análisis de la situación; no obstante, somos una disciplina que se nutre de otras y aprovecha los recursos para el enriquecimiento profesional, por lo que parece interesante incluir este método para valorar en qué punto nos encontramos y hacia dónde nos dirigimos. Es lo que se conoce como "marketing" de los servicios sanitarios [12].

\section{Debilidades}

- En el ámbito asistencial: Dedicación casi exclusiva a una prestación de cuidados orientada en la resolución puntual del problema; Poca conciencia de los profesionales sobre la necesidad de imbricar teoría y práctica; Excesivo predominio del enfoque biomédico tecnicista que impide un mayor desarrollo de nuestra disciplina; Elevada presión asistencial que dificulta la aplicación de planes de cuidados de forma coherente e individualizada; Aumento de la carga asistencial por las excesivas demandas y el aumento poblacional

- En la docencia: Escaso número de profesionales doctorados que puedan impartir docencia a futuros enfermeros; Falta de profesionales enfermeros que puedan ofrecer una formación desde y para la Enfermería 
- En la investigación: Falta de una conciencia investigadora como motor que impulse el crecimiento de la ciencia enfermera; Desconocimiento de los procedimientos a seguir en temas de investigación; Falta de interés o motivación que empujen a iniciar proyectos de investigación; Poca implicación de las administraciones en los proyectos de investigación existentes; Desconexión entre las evidencias investigadas y su aplicación en la praxis; Falta de interés de los propios profesionales que lo consideran como innecesario

- En la gestión: Falta de un mayor desarrollo de la figura del enfermero como gestor de los cuidados de salud del individuo; Falta de concienciación de los propios profesionales sobre qué es y qué nos permite la gestión

\section{Amenazas}

- En el ámbito asistencial: Convivencia en la práctica diaria de tres figuras como prestadoras de cuidados, con perspectivas diferentes, como son el ATS, el practicante y el enfermero; Falta de independencia respecto al modelo biomédico clásico; Poca implicación de las instituciones ; Falta de implicación de la clase política en las reivindicaciones de la Enfermería

- En la docencia: Plasmar incorrectamente la filosofía del EEES centrada en el alumno y su aprendizaje significativo; Incomprensión de lo que supone este cambio para la profesión enfermera; Desarrollo incierto del nuevo marco normativo del EEES

- En la investigación:Insuficientes ayudas y subvenciones de las administraciones para el desarrollo de proyectos de investigación

- En la gestión:Poca motivación de los profesionales para emprender tareas y caminos inciertos; Negativa por parte de ciertos colectivos a que la Enfermería se desarrolle en el ámbito de la gestión; Poca implicación de administraciones y estamentos políticos; Poco interés por parte de la clase política de potenciar la Enfermería en este campo

\section{Fortalezas}

- En el ámbito asistencial: Unificación de criterios en la gestión integral del cuidado del paciente; Desarrollo de un marco competencial propio que guía los planes de cuidados; Toma de conciencia de la importancia que tiene el proceso de comunicación con el paciente y su familia en el desarrollo del plan de cuidados; Manejo de sistemas de registro de enfermería elaborados y validados por enfermeros; Elaboración de protocolos y procedimientos por parte de los propios enfermeros, adaptándolos siempre a la situación real del paciente y su entorno; Concienciación sobre la importancia de la formación continuada como herramienta de actualización constante en una ciencia que cambia a una velocidad vertiginosa

- En el ámbito docente: Se ha conseguido una Enfermería universitaria de alto nivel, y son cada vez más los enfermeros que contribuyen a la formación de futuros profesionales; Desarrollo de las especialidades de Enfermería que persiguen una visión específica de ciertas situaciones sin olvidar el holismo en los cuidados

- En la investigación: Aumento considerable en las publicaciones por parte del profesional de Enfermería; Concienciación de la importancia que tiene la investigación en el desarrollo de la disciplina

- En la gestión: La dirección de Enfermería de los centros se encuentran al mismo nivel de discusión en las Comisiones de Dirección que los directores médicos o administrativos; Modernización de los métodos de trabajo que posibilitan la formación de profesionales capaces de realizar los cambios, impulsar políticas de calidad,... 


\section{Oportunidades}

- En el ámbito asistencial: Se debe implicar al paciente en su proceso de salud, haciéndole partícipe de su propio plan de cuidados; Consideración de la comunicación como herramienta básica que debe guiar la práctica enfermera en la relación con el paciente y su entorno, por lo que se hace necesario el desarrollo de capacidades que mejoren el trabajo en grupo; Integración efectiva y real de un marco conceptual en la práctica enfermera que vertebre la prestación de un plan de cuidados desde el punto de vista holístico

- En la docencia: Implicación de la Enfermería de lleno en la formación de futuros profesionales, para lo cual se precisa aumentar el colectivo de posgraduados que se encaminan a los estudios de doctorado; Incluir aprovechando el nuevo EEES estas inquietudes en el curriculum enfermero, de manera que los estudiantes de enfermería tomen pronto conciencia de la trascendencia de una formación coherente basada en unos evidencias y orientada a la gestión integral del conocimiento

- En la investigación: Evaluación de la rentabilidad de los cuidados enfermeros; Desarrollo y validación de acciones dirigidas a la promoción de la salud; Plena capacidad investigadora que nos otorgará el nuevo EEES

- En la gestión: Diseño, planificación y puesta en marcha de las unidades clínicas de cuidados; Formación correcta y continuada de los profesionales para ofertar una mejor calidad de los cuidados; Mejora de la calidad del cuidado enfermero gracias a la evaluación del mismo

\section{CONCLUSIONES}

Se observa en la actualidad una creciente concienciación sobre las posibilidades de desarrollo de la disciplina enfermera, si bien es cierto que aún es incipiente. Se hace notoria la necesidad de una integración efectiva entre las concepciones o postulados teóricos y sus proyecciones sobre la práctica cotidiana. La Enfermería Basada en la Evidencia surge como una respuesta a estas inquietudes y como una solución que pretende fundamentar las acciones de los profesionales de Enfermería en evidencias objetivables científicamente. El futuro se presenta repleto de retos para nuestra profesión, unos retos que van a vertebrar una Enfermería que no conoce límites. La cuestión estriba en determinar si estamos dispuestos a asumir estos desafíos.

\section{BIBLIOGRAFÍA}

1. Díaz Sánchez V, Álvaro Noguera M, Campo Cecilia E, Saavedra Sánchez F, Cabrera Artacho MD. El paradigma de la Enfermería basada en la evidencia en la enfermería clínica hospitalaria. Nure Inv 2007; 28

2. Zabalegui Yárnoz A. El rol del profesional de Enfermería. Rev. Aquichán 2003; 3(3): 16-20

3. Rivas Riveros E, Montalvo Prieto A, Flórez Torres I. Enfermería para el siglo XXI. Teoría de la Enfermería. ¿Un camino de herradura? Aquichán 2008; 8(1): 6-7

4. Mejía Lopera María Eugenia. Reflexiones sobre la relación interpersonal enfermerapaciente en el ámbito del cuidado clínico. Index Enferm [revista en la Internet]. 2006 Nov [citado 2011 Mar 30] ; 15(54): 48-52. Disponible en: http://scielo.isciii.es/scielo.php?script=sci_arttext\&pid=S113212962006000200010\&ln g=es. doi: $10.4321 /$ S1132-12962006000200010 
5. Silva de Andrade L, Viana L. Reflexiones sobre el paradigma de la complejidad y el caos y sus nexos con la Enfermería del tercer milenio. Enferm Global 2008; 14

6. Suárez B. Importancia de la investigación para el desarrollo dela disciplina de Enfermería. Rev Aquichán 2003; (3): 5-8

7. Entrevista con Callista Roy. Aquichán 2002; 2(2): 36-39

8. Nájera S. Enfermería basada en evidencias. Rev Enferm IMSS 1999; 7(3):145-146

9. Del Pino B. De la teoría a la práctica, un camino difícil en Enfermería. Excel enferm [revista en la Internet]. 2004 Nov [citado 2011 Mar 30] .Disponible en:http://www.ee.isics.es/servlet/Satellite?pagename=ExcelenciaEnfermera/Articulo_E E/plantilla_articulo_EE\&numRevista $=0$ \&idArticulo $=1082026113274$

10.Torralba $\bar{F}$. Enfermería y transformación paradigmática. XX Congreso Nacional de Enfermería de Salud Mental, Alicante 10,11,12 abril 2005

11. Marriner A, Railler M. Modelos y Teorías de Enfermería. Elsevier, Madrid. 2007

12. Murrain E. Tendencias de la investigación en Enfermería. Repert Med Cir 2009; 18 (2): 90-96.

13. Romero B, Nubia M. Enfermería basada en la evidencia. ¿Avanzamos para transformar? Index Enferm. 2003;40-41;41-46

14. Quirós E. El camino de las sombras: Análisis de una experiencia. Nure Invest 2007;31

15.Sanjuán A. Enfermería en la sociedad: Relación teoría/práctica. Cult cuid XI(21): 3340

16.Sanjuán A, Martínez JR. Nuevo enfoque en el proceso de enseñanza-aprendizaje para la interrelación de conocimiento y formación de clínica/comunitaria. Invest Educ enferm 2008; 26 (2 supl): 150-159.

17. Siles J. Las características de los paradigmas y su adecuación a la investigación en Enfermería. Enfermería científica Altoaragonesa 1995; 160-161

18. Siles González, J Epistemología y Enfermería: por una fundamentación científica y profesional de la disciplina. Cuid y Educ 2006 (5): 28-31

19.Zaider V, Sanhuenza O. Paradigmas de investigación en Enfermería. Cienc enferm 2005; XI (1): 17-24

20.San Martin L. Una reflexión en torno a las "ciencias de la enfermería". Enferm clin 2008; 18(5): 262-268

21. Ochoa S. A propósito de la Enfermería basada en la evidencia: algunos cuestionamientos, limitaciones y recomendaciones para su impementación. Invest educ enferm 2005; 23(2): 138-146

ISSN 1695-6141

๑ COPYRIGHT Servicio de Publicaciones - Universidad de Murcia 